\title{
Assessing selfcare status in quadriplegia: comparison of the quadriplegia index of function (QIF) and the functional independence measure (FIM)
}

\author{
R J Marino MD ${ }^{1}$ M Huang MD ${ }^{2}$ P Knight $\mathrm{BS},{ }^{3}$ G J Herbison MD,${ }^{1}$ \\ J F Ditunno Jr MD, ${ }^{1}$ M Segal $\mathrm{PhD}^{1}$ \\ ${ }^{1}$ Department of Rehabilitation Medicine, Thomas Jefferson University Hospital, Suite \\ G9410, 111 South Eleventh Street, Philadelphia, PA 19107, USA; ${ }^{2}$ Jefferson Medical \\ College of Thomas Jefferson University; ${ }^{3}$ New York Medical College, Valhalla, NY.
}

\begin{abstract}
The purpose of this study was to compare the quadriplegia index of function (QIF) to the functional independence measure (FIM) in assessing the daily performance of selfcare activities by individuals with cervical spinal cord injury. This study evaluated feeding, grooming and bathing activities in $22 \mathrm{C} 4-\mathrm{C} 7$, Frankel A-D spinal cord injury patients between 3 and 12 months postinjury. The manual muscle test (MMT) was performed on 17 of these subjects during the same window of time as the QIF and the FIM. An upper extremity motor score (UEMS) was derived from the MMT. In order to relate motor power to functional ability, the UEMS was used as a measure of neurological function to test the hypothesis that the QIF scores are more highly correlated to motor power than are the FIM scores for this population. Spearman coefficients were calculated to correlate the QIF, the FIM and the UEMS. For the bathing and grooming categories, both the QIF and the FIM showed significant and similar correlations to the UEMS. For the feeding category, however, the QIF had a significantly better correlation to the UEMS than did the FIM (Rho $=0.90$ vs $0.53, p<0.01)$. Use of the QIF feeding scale may allow the detection of changes in function as individuals recover that the FIM scale would miss. Further evaluation of the remaining selfcare and mobility scales is needed. Modification of the FIM with more sensitive portions of the QIF would improve the discriminative ability of outcome studies and program evaluations.
\end{abstract}

Keywords: spinal cord injuries; quadriplegia; assessment; selfcare.

\section{Introduction}

A major goal in the rehabilitation of spinal cord injury (SCI) patients is to maximize development of functional skills for activities of daily living. Documentation of the exact extent of functional capacity after SCI is imperative in order to correctly interpret the outcome, ${ }^{1,2}$ cost efficiency, and effectiveness of treatment interventions, and to provide appropriate education for patients and their families. Prognostic information is desired by patients, relatives, employers, and insurance carriers. ${ }^{3}$

It is difficult to predict precisely what activities an individual with cervical SCI will be able to perform after rehabilitation. The difficulty in developing a predictive model of functional capacity in SCI is due in part to a lack of consensus on the appropriate instrument to measure selfcare. Currently, several instruments are being used to measure selfcare function in SCI. The predominant instruments are the modified Barthel index $(\mathrm{MBI}),{ }^{4}$ the functional independence measure (FIM) ${ }^{5}$ and the quadriplegia index of function (QIF). ${ }^{6}$ Of these instruments, only the QIF was designed specifically for use in SCI.

The MBI has been used by several investigators to evaluate subjects in selfcare capabilities. ${ }^{7-8}$ Currently, the FIM is being used by the Model Spinal Cord Injury System supported by the National Institute on Disability and Rehabilitation Research, United States Department of Education. ${ }^{9}$ Roth has shown that the FIM correlates well to the MBI in functional assessment. ${ }^{10}$ 
However, Gresham and associates have stated that the QIF is more sensitive in selfcare evaluation of quadriplegic subjects than the Barthel index. ${ }^{6}$ The QIF and the FIM have not been compared in the literature.

Although the SCI National Data Base uses the FIM, a more sensitive tool such as the QIF may be more appropriate for use in documenting selfcare status in quadriplegic patients. ${ }^{11}$ One of the drawbacks in using FIM is that the score is based upon the level of independence achieved in an entire group of selfcare activities for a specific category. Hence, only a global score for a category is calculated. The QIF, on the other hand, while providing a calculated category score, also scores individual task items within a category. ${ }^{6}$ The QIF scoring of separate items may allow a finer discrimination of functional ability and a more precise description of functional abilities by level of injury.

Previous studies have demonstrated a positive correlation between motor power and functional ability. ${ }^{11-13}$ The motor score has been used in several studies as a measure of neurological status, with changes in motor score over time indicating the degree of recovery. ${ }^{14,15}$ This study relates strength to function by intercorrelating motor score, the QIF and the FIM. In this preliminary study, only the feeding, bathing and grooming categories of the QIF and the FIM are analyzed. Because these activities are performed with the upper extremities, the upper extremity motor score (UEMS) of the manual muscle test (MMT) is employed to assess motor power. The present study tests the hypotheses that the QIF and the FIM differ in the assessment of selfcare function in quadriplegia, and that QIF scores are more highly correlated to motor power than are FIM scores for the categories tested.

\section{Methods}

The subjects were 22 males with traumatic quadriplegia, C4-C7, Frankel A-D, admitted to the SCI center within 72 hours of injury, during the period from November 1989 to March 1991. Ages ranged from 18 to 63 years with a mean age of 33 years. All subjects were medically stable, without acute medical conditions, amputation, stroke, closed head injury, upper extremity fractures, knife or gunshot wounds at the time of evaluation, or evidence of variability in functional performance due to lack of motivation, cooperation, or psychiatric disorders. At the initial (72 hour) examination, the neurological level of injury was $\mathrm{C} 4$ for 6 subjects, C5 for 7, C6 for 4, and C7 for 5 . Four subjects were Frankel A, 11 were Frankel B, 2 were Frankel C, and 5 were Frankel D.

As part of ongoing studies, subjects were assessed by MMT and the QIF at 3, 6 and 12 months postinjury. The FIM was administered at admission to and discharge from rehabilitation. These assessments were performed by interview, and evaluated what the individual was doing on a regular basis at the time of assessment. To be included in this study, subjects needed to have both a QIF and a FIM within one of the following time windows: 3 months post SCI plus or minus one week, 6 months post SCI less one week or plus one month, and 12 months post SCI plus or minus two months. Of the 22 subjects in the study, 7 were evaluated at 3 months, 10 at 6 months, and 5 at 12 months.

In addition, 17 of the subjects had a MMT performed within the same window of time as the QIF and FIM, and had at least one biceps that was greater than $3 / 5$ by MMT. These subjects had intercorrelations of MMT, QIF and FIM. At the time of testing for this group, 3 subjects were $\mathrm{C} 4$ neurological level, 5 were $\mathrm{C} 5,4$ were $\mathrm{C} 6$, and 5 were C7. The Frankel grades were as follows: 3 Frankel A, 7 Frankel B, 2 Frankel $\mathrm{C}$, and 5 Frankel D.

The MMT is used by the American Spinal Injury Association to evaluate the motor function of patients following SCI and during rehabilitation. ${ }^{16}$ The motor function for individual muscle groups is scored on a scale of $0-5$ as follows: 0 -absent (total paralysis); 1 -trace (palpable or visible contraction); 2-poor (active movement through full range of motion (ROM) with gravity eliminated); 3-fair (active movement through full ROM against gravity); 4-good 
(active movement through full ROM against resistance); 5-normal.

The biceps, wrist extensors, triceps, flexor digitorum profundus, and hand interossei were tested bilaterally. An upper extremity motor score (UEMS) was obtained by summing the individual muscle group scores of the left and right upper extremities. This differs from the total MMT score which sums the muscle grades for key muscles in both the upper and lower extremities. The UEMS was selected because the selfcare tasks analyzed require primarily upper extremity function to complete the activity. Use of the UEMS allows inclusion of both complete and incomplete patients in the same group.

The QIF was designed to assess the functional ability of quadriplegic patients and to monitor their progress in rehabilitation. ${ }^{6}$ The QIF consists of two parts. The first part assesses specific activities grouped into nine categories: transfers, grooming, bathing, feeding, dressing, wheelchair mobility, bed activities, bowel program, and bladder program. The second part consists of a supplemental questionnaire that assesses the patient's understanding of personal care. Each category contains several items that are scored from $0-4$ in order of increasing independence (Table I). Category scores are obtained by summing the item scores in a particular category. The category and questionnaire scores are then weighted. These weighted scores are

Table I Scoring of selfcare tasks

\begin{tabular}{|c|c|c|c|}
\hline \multicolumn{2}{|l|}{ QIF items } & \multicolumn{2}{|c|}{ FIM categories } \\
\hline 4 & Independent & & 7 \\
\hline 3 & $\begin{array}{l}\text { Independent with } \\
\text { devices }\end{array}$ & & 6 \\
\hline 2 & Supervision & & 5 \\
\hline & & Min & 4 \\
\hline 1 & Physical assistance & Mod & 3 \\
\hline & & Max & 2 \\
\hline 0 & Dependent & & 1 \\
\hline
\end{tabular}

$\mathrm{QIF}=$ quadriplegia index of function

$\mathrm{FIM}=$ functional independence measure

Min $=$ minimal

Mod $=$ moderate

Max $=$ maximal summed to calculate a QIF score ranging from $0-100$.

The present study was limited to the QIF items in three selfcare categories: grooming, bathing and feeding (partial QIF). A total of 14 items were scored for the partial QIF within the three categories tested (Table II). Category scores were obtained by summing the individual items within each category. For example, a patient that scored 4 on brushing teeth, 3 on brushing hair, and 3 on shaving would receive a QIFgroom score of 10. These categorical scores will be referred to as the QIFbath, QIFgroom, and QIFfeed scores. A partial QIF score was then calculated by adding the three categorical scores. Note that this study employed unweighted categorical scores to factilitate analysis of each category when comparing it to the respective FIM categories.

The FIM also assesses a patient's selfcare status. ${ }^{5}$ The selfcare group consists of six items: eating, grooming, bathing, dressing/ upper body, dressing/lower body, and toileting. The FIM items represent major divisions of the FIM much as the QIF categories represent major divisions of the QIF. This study will refer to the FIM items as FIM categories to remain consistent with QIF terminology.

Table II QIF categories and items

\begin{tabular}{ll}
\hline Category & Item \\
\hline Grooming & \\
& $\begin{array}{l}\text { Groom 1: Brushing teeth/ } \\
\text { managing dentures } \\
\text { Groom 2: Brushing/combing hair } \\
\text { Groom 3: Shaving (for men) }\end{array}$
\end{tabular}

Bathing

Bath 1: Wash/dry upper body Bath 2: Wash/dry lower body Bath 3: Wash/dry feet Bath 4: Wash/dry hair

Feeding

Feed 1: Drink from cup/glass

Feed 2: Use spoon/fork

Feed 3: Cut food (meat)

Feed 4: Pour liquids

Feed 5: Open carton/jar

Feed 6: Apply spreads to bread

Feed 7: Prepare simple meals 
For this study, the FIM categories of grooming, bathing, and eating were scored for each subject. The FIM is scored in each category on a seven point scale in order of increasing independence (Table I). Performance in the category as a whole is the basis of the scoring, rather than performance in individual components of the activity. Thus, only global category scores are received on the FIM. For example, a patient must be able to perform all defined grooming activities with supervision in order to receive a FIMgroom score of 5 . The categorical scores will be referred to as the FIMgroom, FIMbath, FIMfeed. The partial FIM score was obtained by summing the categorical scores.

The raw frequencies and percentages of scores for the partial QIF, partial FIM, QIF categorical scores, and FIM categorical scores were determined, and the distributions of the QIF scores and the FIM scores were compared. Spearman correlation coefficients were calculated for the following: (1) UEMS score to the partial QIF score and the partial FIM score; (2) UEMS score to the QIF categorical scores; (3) UEMS score to the FIM categorical scores; (4) partial QIF score to partial FIM score; (5) QIF categorical scores to FIM categorical scores; (6) intercorrelation of QIF grooming items; (7) intercorrelation of QIF bathing items; and (8) intercorrelation of QIF feeding items. Spearman correlation coefficients were compared for significant differences using a standard formula, ${ }^{17}$ with significance levels set at 0.05 .

To simplify comparisons of Spearman correlation coefficients the following designations were used: $0.00-0.49=$ poor correlation; $0.50-0.69=$ fair correlation; 0.70 $0.84=$ good correlation; $0.85-1.00=$ excellent correlation. These divisions were arbitrary, as no strict statistical definitions exist for degree of correlation with Spearman values.

\section{Results}

Scatter diagrams plotting the partial QIF score versus UEMS (Fig 1) or partial FIM score versus UEMS (Fig 2) demonstrate the relationship between the functional assess-

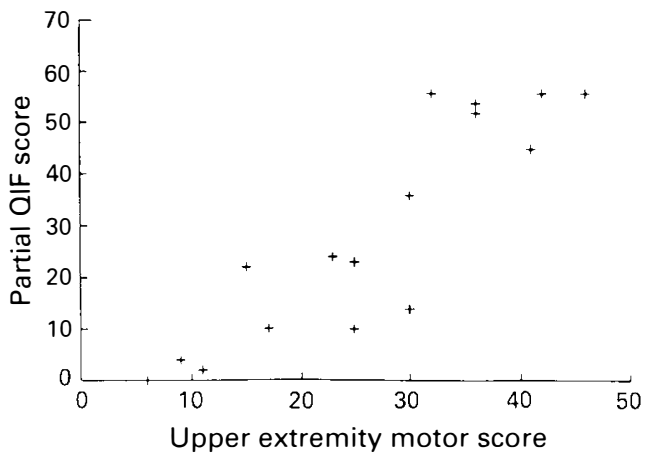

Figure 1 Scatterplot of upper extremity motor score (UEMS) versus partial quadriplegia index of function (QIF) score; Spearman Rho $=0.91$.

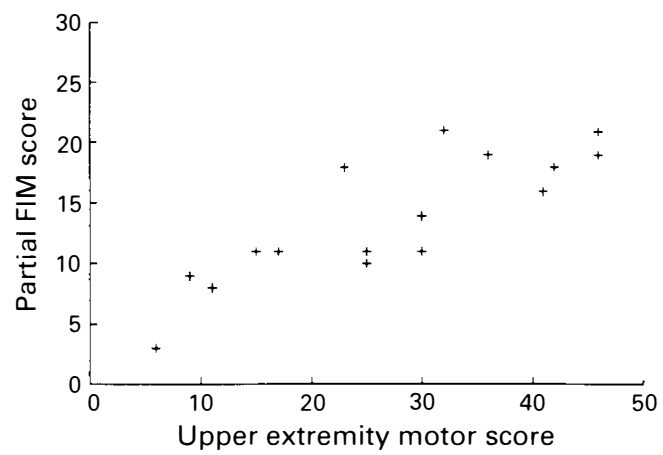

Figure 2 Scatterplot of upper extremity motor score (UEMS) versus partial functional independence measure (FIM) score; Spearman Rho $=0.84$.

ment tests and motor power. The QIF has an excellent and the FIM a good correlation to the UEMS score, with correlation coefficients of 0.91 and 0.84 respectively. The difference between the QIF and FIM correlations to UEMS was not statistically significant.

The results of QIF and FIM intercorrelations, as well as intercorrelations between the UEMS and the QIF or the FIM, are shown in Table III. The correlation between feeding scores was good, but lower than correlations between the other categories tested. Feeding ability assessed by the QIF was significantly better related to motor power than feeding ability assessed by the FIM $(p<.01)$. All other comparisons were not significantly different. 
Table III Spearmen correlations (Rho) of QIF and FIM and the UEMS

\begin{tabular}{llll}
\hline & $\begin{array}{c}\text { UEMS } \\
\text { vs } \\
\text { QIF }\end{array}$ & $\begin{array}{c}\text { UEMS } \\
\text { vs } \\
\text { FIM }\end{array}$ & $\begin{array}{c}\text { QIF } \\
\text { vs } \\
\text { FIM }\end{array}$ \\
\hline Grooming & 0.90 & 0.91 & 0.94 \\
Bathing & 0.84 & 0.75 & 0.92 \\
Feeding & $0.90^{*}$ & $0.53^{*}$ & 0.75 \\
Partial & 0.91 & 0.84 & 0.93 \\
\hline
\end{tabular}

QIF = quadriplegia index of function

FIM = functional independence measure

UEMS = upper extremity motor score

${ }^{*} p<0.01$

The distribution of scores for the partial QIF and FIM displayed some differences. The QIF distributed the 22 subjects over 17 scores from 0-56, while the FIM distributed subjects over 12 scores ranging from 3-21. The partial FIM showed clustering of subjects around the scores of 11,19 and 21 with $23 \%, 14 \%$ and $14 \%$ of the subjects obtaining each score respectively. The QIF displayed fairly even distribution with clustering only at the maximum score of 56 ( $23 \%$ of subjects).

It is the feeding category that accounts for most of the discrepancy between the QIF and FIM distributions. The QIF and the FIM grooming and bathing categories possess similar distributions of scores, with subjects distributed over seven scores in both scales. Conversely, the distribution of scores in the feeding category differed markedly between the QIFfeed and FIMfeed (Table IV). The QIFfeed distributed subjects over 13 different scores while only five separate scores were obtained on the FIMfeed.

Intercorrelations between QIF items within the same category were calculated for grooming, bathing, and feeding (Table V). The Spearman correlations for grooming items ranged from 0.65 to 0.70 , for bathing items from 0.67 to 0.93 , and for feeding items from 0.44 to 0.88 .

Correlations between the QIF and the FIM using only the 17 subjects with motor scores were performed and were not meaningfully different from correlations using the entire sample.

\section{Discussion}

The results of this study partially support the hypothesis that the QIF differs from the

Table IV Distribution of scores for the category of feeding $(n=22)$

\begin{tabular}{|c|c|c|c|c|c|}
\hline \multicolumn{3}{|c|}{ QIF feed } & \multicolumn{3}{|c|}{ FIM feed } \\
\hline Score & Freq & Percent & Score & Freq & Percent \\
\hline 0 & 1 & 4.5 & 1 & 1 & 4.5 \\
\hline 2 & 1 & 4.5 & & & \\
\hline 3 & 1 & 4.5 & & & \\
\hline 4 & 1 & 4.5 & 4 & 3 & 13.6 \\
\hline 6 & 2 & 9.1 & & & \\
\hline 7 & 2 & 9.1 & & & \\
\hline 8 & 1 & 4.5 & & & \\
\hline 9 & 1 & 4.5 & 5 & 9 & 40.9 \\
\hline 14 & 1 & 4.5 & & & \\
\hline 19 & 1 & 4.5 & & & \\
\hline 20 & 2 & 9.1 & & & \\
\hline 24 & 1 & 4.5 & 6 & 3 & 13.6 \\
\hline 26 & 1 & 4.5 & & & \\
\hline 28 & 6 & 27.3 & 7 & 6 & 27.3 \\
\hline
\end{tabular}

$\mathrm{QIF}=$ quadriplegia index of function

$\mathrm{FIM}=$ functional independence measure

Freq $=$ frequency 
Table $\mathbf{V}$ Spearman correlation coefficients of category items of the quadriplegia index function $(n=22)$

Intercorrelation of bathing items

Bath 1

Bath 2

Bath 3

Intercorrelation of grooming items

Groom 1

Groom 2

Intercorrelation of feeding items

Feed 1

Feed 2

Feed 2

Feed 3

Feed 4

Feed 5

Feed 6

$\begin{array}{ccc}\text { Bath 2 } & \text { Bath 3 } & \text { Bath 4 } \\ 0.86 & 0.67 & 0.70 \\ & 0.93 & 0.75 \\ & & 0.79\end{array}$

$\begin{array}{cc}\text { Groom } 2 & \text { Groom } 3 \\ 0.70 & 0.65 \\ & 0.67\end{array}$

$\begin{array}{cc}\text { Feed } 3 & \text { Feed } 4 \\ 0.44 & 0.59 \\ 0.66 & 0.88 \\ & 0.75\end{array}$

Feed 5

0.44

0.66

0.77

0.75

$\begin{array}{cc}\text { Feed } 6 & \text { Feed } 7 \\ 0.53 & 0.48 \\ 0.71 & 0.72 \\ 0.68 & 0.56 \\ 0.80 & 0.82 \\ 0.76 & 0.84 \\ & 0.85\end{array}$

FIM in assessment of selfcare performance and that the QIF is more highly related to motor power. Overall, both the partial QIF and partial FIM showed a good correlation with the UEMS (Table III). There was a trend towards the superiority of the partial QIF; however, the difference between the partial QIF and partial FIM was not significant. When looking at the categories of the two scales, the category of feeding produced a major and statistically significant difference between the QIF and the FIM. This accounted for most of the difference between the partial QIF and partial FIM. The feeding categories of the two scales did not correlate as well with each other, unlike the bathing and grooming categories. Furthermore, it was the QIFfeed, not the FIMfeed, which displayed the best correlation with the UEMS.

An examination of the intercorrelation of items in the QIF categories and the scoring method of the two scales may account for the difference found in the feeding component. The FIM, as mentioned before, assigns only one global score per category to a subject, while the QIF scores each task within the category. This may yield a difference between the scales if the task items within a category are not of comparable difficulty. In other words, highly correlated items are those for which knowing the score on one item will allow an accurate prediction of the score on the other. Little discriminative information is gained by scoring these items separately. Poorly correlated items imply items of disparate difficulty. The score on one item has little relation to the score on the other. In this case, scoring each item individually would yield more information than an isolated global score.

The QIF feeding category has several items which correlate poorly, particularly 'drinking from a cup'; 'cutting foods', 'opening containers', 'applying spreads', 'pouring liquids', and 'preparing simple meals'. Thus, the QIFfeed items are not comparable. The bathing and grooming items, on the other hand, are much more comparable. There are only three items in grooming, and they are all moderately related. While there are four bathing items, there is little difference between 'wash/dry lower body' and 'wash/ dry feet' (rho $=0.93$ ), and the remaining items have fair correlations. Thus the feeding category has the greatest number of disparate items, and would stand to gain the most from individual item scoring. It is not surprising, then, that the QIFfeed and FIMfeed were only moderately correlated, while the grooming and bathing categories of the scale were highly correlated. 
An examination of the distribution of scores for feeding by the QIF and the FIM highlights a difference between the two scales, and the drawback of global category scoring. The FIM displayed clustering around both the maximum score and a score of 5, while the QIF displayed clustering only around the maximum score (Table IV). At the low and high ends, both scales are similar. However, the clustering of scores around 5 for FIMfeed suggests that it assigns the same score to subjects possessing different functional levels. FIMfeed defines 5 as patients who need supervision, coaxing, or help applying a prosthesis to perform numerous 'set up' tasks including cutting food, pouring liquids, buttering bread, and opening containers. This definition is too broad as it classifies subjects who are able to perform some but not all of these tasks independently at the same functional level as those who need help with all the tasks. These FIM 'set up' items correspond to items 3-6 of the QIFfeed. As was discussed above, these items are poorly correlated with other items of the QIFfeed, particularly, with 'drink from cup/glass', and are only moderately intercorrelated. Therefore, being able to accomplish one of these tasks does not mean a subject will be able to complete the others. The QIF scores each item, so that progress on any of the four tasks is reflected in the total category score. The FIM scoring, on the other hand, requires the subject to be able to perform all set up tasks before reaching the next level (supervision, score of 6).

Comparing the QIFgroom to the FIMgroom and the QIFbath to the FIMbath, one finds that the distribution of scores was comparable; clustering occurred at the maximum score with a fairly even distribution below that score. Because several subjects were Frankel D at the time of testing, and subjects were tested at the end of their rehabilitation stay, they would be expected to perform well on the selfcare assessments. Therefore the clustering of scores at the maximum of the categories is not surprising. The similar distribution of scores implies comparable scoring by the two tests.

This study has several limitations. Because of the small number of subjects, the power of the comparisons is low, and small statistically significant differences between the scales may have been missed. The QIFgroom and QIFbath have few items relative to the QIFfeed, and would require evaluating more subjects to identify a difference in scoring with the FIM. The numbers of subjects were too small to separate complete from incomplete subjects. Complete patients may have differences in function that would be best differentiated by the QIF. ${ }^{18}$ The timing of the motor and functional assessments was not identical, so that changes in function or strength between assessments may have affected the results. The size of the windows for the assessment periods were chosen to minimize this possibility. Finally, only a portion of the QIF and the FIM was compared. If differences similar to those found in the feeding category are found on comparing other categories, then these differences may be enough to achieve significant differences on the scales as a whole.

The QIF scoring of individual items allows examiners to assess a patient's ability on individual tasks, something the FIM's global scoring does not permit. This can be important in terms of that individual's rehabilitation program. The QIF may be able to identify an improvement in functional ability whereas the FIM score would not reflect a change in ability. This is especially true for some subsets of quadriplegic patients who may make small significant gains. ${ }^{19,20}$

Recent interventional studies in acute SCI have demonstrated small, statistically significant gains in motor power with the use of methylprednisolone ${ }^{14}$ and GM-1 gangliosides. ${ }^{15}$ No functional assessment measure was included in these studies, leaving one to wonder about the functional significance of the increased motor power. The current National Acute Spinal Cord Injury Study trial has included the FIM as part of its outcome data. However, if the FIM is not sensitive enough, it may miss real changes in functional status. This study suggests that the QIF is more sensitive than the FIM in the assessment of feeding. Further study comparing the remaining selfcare items and the mobility items is indicated to determine 
if there are other areas of relative insensitivity in the FIM.

Modifications of some categories of the FIM may be desirable in research studies evaluating functional outcomes in quadriplegia. The FIM assessment requires 20-30 minutes to complete in its entirety. The total QIF requires approximately 15-20 minutes. Substituting certain portions of the QIF for portions of the FIM could increase the sensitivity of the tool without adding to the time of evaluation.

In conclusion, when related to motor power the QIF assessed functional ability in the category of feeding more accurately than did the FIM. The categories of grooming and bathing were comparable, with a trend towards QIFbath showing a higher correlation than FIMbath. A larger prospective study involving 80 to 100 subjects seems warranted. This would remedy the weaknesses of this study by increasing the power, allowing comparison of complete and incomplete quadriplegics, and standardizing the time of assessment. Further study should lead to a better understanding of how these two tests assess functional ability, and whether other categories of the QIF have advantages over the FIM in relating motor power to function.

\section{Acknowledgements}

This study was supported in part by awards from the National Institute on Disability and Rehabilitation Research to the Regional Spinal Cord Injury Center of Delaware Valley (\#H133N00027) and the National Rehabilitation Research and Training Center in Spinal Cord Injury (\#H133B80017).

\section{References}

1 Heinemann A, Yarkony G, Roth E et al (1989) Functional outcome following spinal cord injury. A comparison of specialized spinal cord injury center vs. general hospital short-term care. Arch Neurol 46: 1098-1102.

2 Cole TM, Egerton V (1990) Report of the Task Force on Medical Rehabilitation Research, Hunt Valley, Maryland: June 28-29, 1990: 43-56.

3 Meinecke F (1985) Some thoughts about neurologic recovery in spinal cord injuries: a philosophical review. Paraplegia 23: 78-81.

4 Granger CV, Albrecht GL, Hamilton BB (1979) Outcome of comprehensive medical rehabilitation: measurement by PULSES profile and the Barthel index. Arch Phys Med Rehabil 60: 145-154.

5 Uniform Data Set for Medical Rehabilitation Version 3.0 March 1990. Data Management Service of the Uniform Data System for Medical Rehabilitation and the Center for Functional Assessment Research. SUNY Buffalo, 82 Fuher Hall; SUNY Main St, Buffalo, NY 14214.

6 Gresham GE, Labi M, Dittmar S, Hicks J, Joyce S, Phillips Stehlik M (1986) The quadriplegic index of function (QIF): sensitivity and reliability demonstrated in a study of thirty quadriplegic patients. Paraplegia 24: 38-44.

7 Yarkony G, Roth E, Lovell L, Heinemann A, Katz R, Wu Y (1988) Rehabilitation outcomes in complete C5 quadriplegia. Am J Phys Med Rehabil 1: 73-76.

8 Yarkony G, Roth E, Heinemann A, Lovell L (1990) Rehabilitation outcomes in C6 tetraplegia. Paraplegia 26: $177-185$.

9 Stover S (1990) National Spinal Cord Injury Statistical Center Annual Report No 8. University of Alabama at Birmingham, Oct 1989 through Sept 1990: 83: 93-94.

10 Roth E, Davidoff G, Haughton J, Ardner M (1990) Functional assessment in spinal cord injury: a comparison of the modified Barthel index and the 'adapted' functional independence measure. Clin Rehabil 4: $277-285$.

11 Ditunno J, Graziani V (1989) Motor recovery and functional prognosis in spinal cord injury. Rehabil Report 5: $1-4$.

12 Welch R, Lobley S, O'Sullivan S, Freed M (1986) Functional independence in quadriplegia: critical levels. Arch Phys Med Rehabil 67: 235-240.

13 Lazar R, Yarkony G, Ortolano D, Heinemann A, Perlow E, Lovell L et al (1989) Prediction of functional outcome by motor capability after spinal cord injury. Arch Phys Med Rehabil 70: 819-822.

14 Bracken MB, Shepard MJ, Collins WF, Holford TR, Young W, Baskin DS, Eisenberg HM et al (1990) A randomized, controlled trial of methylprednisolone or naloxone in the treatment of acute spinal-cord injury: results of the Second National Acute Spinal Cord Injury Study. N Engl J Med 322: 1405-1411.

15 Geisler FH, Dorsey FC, Coleman WP (1991) Recovery of motor function after spinal-cord injury - a randomized, placebo-controlled trial with GM-1 ganglioside. N Engl J Med 324: 1829-1838. 
16 American Spinal Injury Association (1992) Standards for neurological and functional classification of spinal cord injury, revised 1992. ASIA, Chicago: 1-26.

17 Walker H, Joseph L (1953) Statistical Inference. Holt, Rinehart, and Winston, NY: 256-257.

18 Zafonte RD, Demangone DA, Herbison GJ, Ditunno JF Jr (1991) Daily self-care in quadriplegic subjects. Neuro Rehabil 1(4): 17-24.

19 Yarkony G, Roth E, Heinemann A, Lovell L, Wu Y (1988) Functional skills after spinal cord injury rehabilitation: three year longitudinal follow-up. Arch Phys Med Rehabil 69: 111-114.

20 Yarkony G, Roth E, Heinemann A, Wu Y, Katz R, Lovell L (1987) Benefits of rehabilitation for traumatic spinal cord injury. Multivariate analysis in 711 patients. Arch Neurol 44: 93-96. 